\title{
LASER REMOTE SENSORS FOR NASA'S FUTURE EARTH AND SPACE SCIENCE MISSIONS
}

\author{
Upendra N. Singh \\ NASA Engineering Safety Center, NASA Langley Research Center, Hampton, Virginia 23681, USA \\ *Email: Upendra.n.singh@nasa.gov
}

\begin{abstract}
Active optical (Laser/Lidar) measurement techniques are critical for the future National Aeronautics and Space Administration (NASA) Earth, Planetary Science, Exploration, and Aeronautics measurements. The latest science decadal surveys recommend a number of missions requiring active optical systems to meet the science measurement objectives and the aeronautics community continues to use Laser/Lidar technologies to meet the aeronautics measurement objectives. This presentation will provide an overview of NASA efforts in developing and maturing state-of-the-art advanced solid-state flight laser/lidar systems for airborne and space-borne remote sensing measurements. The presentation will also provide details of a strategic approach for active optical technologies and techniques to meet the NASA's future Earth and Space Science measurements/missions needs and requirements for space-based applications.
\end{abstract}

\section{OVERVIEW}

NASA's Strategic Plan calls for NASA "to improve prediction of climate, weather, and natural hazards," and to provide services to the Nation including "weather forecasting; climate prediction; natural hazard assessment, prediction, and response; and environmental management, including air quality forecasting and land use assessment." These assignments will require the use of Light Detection And Ranging (lidar) (or laser radar) remote sensing systems. Lidar sensors provide the excellent vertical resolution needed to do these jobs. NASA also is working to advance radar, laser, and light detection and ranging technologies to enable monitoring of such key Earth system parameters as land surface, oceans, ice sheet topography, and global tropospheric winds that could lead to advances in weather and severe storm prediction. After 35 years of passive and then microwave radar remote sensing, NASA began to utilize lidar sensors in the 1990's. However, many difficulties were encountered with the lidar missions. Part of the cause of these difficulties arose from the large gap between the technology maturities after Instrument Incubator Program (IIP) completion (
$\$ 3 \mathrm{M})$, and the desired maturities to begin an Earth System Science Pathfinder (ESSP) flight projects ( $\$ 300 \mathrm{M})$. Too often, the laser and lidar technologies were still being developed during the flight projects, leading to schedule delays and large labor bills from the project personnel. These concerns led to NASA commissioning an Earth Science Independent Laser Review Panel during the early part of 2000. The Panel report cited critical deficiencies in NASA's laser strategy and recommended that NASA should address the cited deficiencies and put together a Laser Risk Reduction Program [1]. Through the Panel report, it was clear that development teams needed to mature laser and lidar technologies before the approval of flight projects. For this reason, NASA approved the Laser Risk Reduction Program (LRRP), which began in 2002, had enjoyed many successes, and ended during 2010 [2-3].

\section{RATIONALE FOR ACTIVE OPTICAL SENSING}

Many physical phenomena critical to improving our understanding of Earth and planetary systems can only be measured practically using lidar techniques. Lidar remote sensing is analogous to radar remote sensing, except lidar employs electromagnetic radiation with wavelengths 4 to 5 orders of magnitude shorter than radar. This wavelength difference causes proportionally less beam divergence; profound differences in usable targets, atmospheric transmission, and attainable resolution. Lidar also requires different beam generation, handling, and detection technologies. Therefore, lidar sensors maintain the advantages of active radar sensors with the self-contained light source, choice and knowledge of exciting signal properties, pointing at desired target, and range-resolved return signal, while providing greatly improved horizontal and vertical spatial resolution, and access to new atmospheric and surface targets. In summary, lidar sensors offer:

- Excellent vertical and horizontal spatial resolution

- Relative independence from natural light sources, their angles, and time of day

- Choice of optical wavelength, pulse duration, pulse shape, and polarization to optimize science return (i.e., the ability to target specific chemical and physical phenomena by choosing the appropriate wavelength)

- Choice of pointing direction 
- Choice of several lidar techniques: basic, altimetry, Doppler, DIfferential Absorption Lidar (DIAL), and polarization

An active optical remote sensing technology development and maturation strategic approach will mitigate lidar system technology development risks by coordinating activities with multiple NASA Centers and government agencies. This will move NASA into the next logical era of lidar remote sensing by enabling such critical space-based measurements as winds, ozone, $\mathrm{CO}$, moisture, clouds, aerosols, surface topography, ice thickness, vegetation, trace atmospheric species, biogenic traces gases and materials, and oceanic properties.

Lidar is also crucial to improving the safety, reliability, and affordability of solar system exploration by providing atmospheric information for spacecraft aerocapture, entry and descent; and guidance and hazard avoidance data to the Planetary Landers. Furthermore, lidar techniques are also regarded as enabling for a variety of homeland security, aviation, and environmental pollution applications. NASA's leadership in this area would further cement its public perception as a national asset and "crossover" entity encompassing both civil and DoD functions. This effort will bring all lidar remote sensing technologies together, including planetary, earth viewing, aerospace, and aeronautics.

\section{KEY MEASUREMENTS ENABLED}

An active optical strategic approach enables several key measurements that are likely to open new frontiers of research that practitioners cannot obtain in any other way. Especially critical measurements from the Earth Science measurement roadmaps include:

- Global tropospheric winds, which are acknowledged as the greatest unmet observational need for improving global weather forecasts on anything more than an incremental basis. Doppler lidar techniques are recognized as the only means for acquiring such data with the required precision $(1 \mathrm{~m} / \mathrm{s}, 25-100-\mathrm{km}$ horizontal resolution).

- Similarly, the location, quantification, and extent of $\mathrm{CO} 2$ sources and sinks; and their variability are prima facie requirements for balancing the carbon budget in global climate models. High precision profiling of tropospheric $\mathrm{CO} 2(0.3 \%$ mixing ratio, $2-\mathrm{km}$ vertical scale) is essential to understanding the global carbon cycle, greenhouse warming and sustainability of life on
Earth can only be obtained through the use of differential absorption lidar (DIAL) lidar techniques.

- Measurement of the global distributions of tropospheric ozone and aerosols using a space-based DIAL system will help address the causes and impacts of long-term climate variability and will distinguish natural from human-induced drivers. This measurement is essential to the modeling and assessment of chemical, radiative, convective, dynamical, and transport processes in the troposphere. Again, precise, vertical measurements (1 to $2 \mathrm{~km}$ vertical) are only available through the use of lidar techniques.

- Lidar is the only method for measuring particle profiles in the oceans' mixing layer, necessary to understand how oceanic carbon storage and fluxes contribute to the global carbon cycle

- DIAL lidar is the only technique for global moisture profiles at high resolution $(0.5 \mathrm{~km}$ vertical by $50 \mathrm{~km}$ horizontal) in the boundary layer. This is an essential need for understanding the convective processes and severe storm development, and to improve our understanding of the global hydrological and energy cycle.

- Backscatter lidar is the only method for high vertical resolution $(30 \mathrm{~m})$ measurements of optical properties of clouds and aerosols including planetary boundary height, cloud base, cloud top, cloud amount, cloud depolarization, and aerosol microphysical properties needed in climate modeling and research.

- Altimetry lidar is the only technique for directly profiling a surface level changes of less than $1 \mathrm{~cm} /$ year, essential for studying terrestrial land cover vegetation, land surface topography, volcano monitoring, global sea level, and polar ice sheet level changes caused by climatic changes.

Especially critical measurements from the Space Science measurement roadmaps include:

- Lidar techniques offer versatile planetary surface topography measurements and other planetary surface deformation information with sub-meter vertical accuracy laser altimetry to study geological, volcanic, climatic, and tidal effects in planetary bodies including Mars, the Moon, Venus, Titan, and Jupiter's Icy Moons. - As with terrestrial applications, backscatter lidar is the only method for determining detailed vertical profiles of the Martian and Jovian atmospheres including dust storms and surface eddies that can provide information about their aerosol and clouds needed to study the structure, dynamical, and radiative properties of the atmospheres of these planetary bodies. - DIAL lidar enables measurement of the variability of water vapor, ozone, $\mathrm{CO}$, and other trace gas species in planetary atmospheres (e.g., Mars) to study the chemical and radiative properties and obtain 
information about water and water vapor that are of keen interest as an indispensable ingredient for life.

- Doppler wind lidar is the only technique to study the three-dimensional dynamical and transport properties, dust storms, and turbulence in the atmospheres of Mars, Jupiter, Venus, Titan, and other planetary systems that can shed new insight into knowledge about the state of these atmospheres

- Surface-based lidar is ideal for measuring tropospheric winds on Mars as a boundary condition in global climate models, but also to ensure design safety of next-generation entry, descent, and landing systems.

- Lidar with high power lasers can be used as remote sensing mass spectrometers by performing laser ablation of distant planetary bodies including Jupiter's Icy Moons, as well as direct compositional information about primitive objects including asteroids and comet nuclei.

- Lidar Raman Spectroscopy for detection of organic phases at local and remote scales (e.g., on Mars)

- Lidar spectroscopy for Biogenic Trace Gas sensing (i.e., detection of methane and other species) in the Martian atmosphere.

\section{ACTIVE OPTICAL CAPABILITY ASSESSMENT AND STRATEGY}

NASA Sensors and Instrumentation (S\&I) Capability Leadership Team (CLT) was recently tasked by the NASA Agency Program Management Council (APMC) to determine if the Agency has the necessary expertise and capabilities to execute successfully the active optical-based systems necessary to make the required measurements for Science, Exploration, and Aeronautics. Given the charter from the APMC, the S\&I CLT leadership assembled a core team to realize the assessment. The Active Optical assessment process utilized a multi-directorate teaming with representatives of the Science, Human Exploration and Operations, Aeronautics Research, and Space Technology Mission Directorates to provide guidance and list the priority measurements requirements to meet their critical future needs.

An Active Optical Tiger team consisting of mission directorate representatives, S\&I capability and technical leads/co-leads, and an external assessment team worked together to seek national and international community inputs to formulate an integrated Agency level strategy. Given the cross-cutting synergies in critical Active Optical measurements for NASA Science, Exploration and Aeronautics, a strategic approach is needed to identify areas where NASA should "lead, leverage or collaborate" with existing national and international industries to meet its future needs. An essential part of the assessment strategy was to engage with other US Government entities, as well as industry and academia, to leverage common Active Optical interests and complementary skills/expertise resident in those sectors. To seek the input from broader national entities, such as NASA, industry, academia, Federally Funded Research and Development Centers (FFRDC) and Other Government Agencies (OGA), the S\&I CLT organized a Technical Interchange Meeting (TIM) during July 31 through August 2, 2018 in Columbia, Maryland, USA. Participants in the TIM exchanged perspectives on the current state of the discipline's technologies and the direction NASA needs to take in the future to raise the TRL of the measurement technologies to meet these measurement needs in the applications domains. The TIM aimed at focusing NASA's directions to attain the necessary technology TRL levels to meet the Agency-level priority Active Optical measurements in Space and Aeronautics.

\section{PRIORITY ACTIVE OPTICAL BASED MEASUREMENT AREAS}

The NASA TIM organized focused technical sessions in the priority active-optical based measurements area for NASA critical needs in Science, Exploration, and Aeronautics. These sessions were:

\subsection{Mission Area I: Earth Science Focused Measurement Topic Areas}

Atmospheric Composition sounding measurements in the troposphere including aerosol and cloud properties and profiling, water vapor profiling, and Planetary Boundary Layer height

- Earth's Surface \& Interior via geodetic imaging including wide-swath laser altimetry

- Climate Effect Monitoring including ice mass changes, global ice characterization, snow depth, and snow-water equivalent measurements

- Weather including global tropospheric Lidar wind measurements for planetary boundary layer energy, momentum, and mass exchanges; forecasting; convection; fluxes of heat, 
momentum, water vapor and other gases; and model validation and improvement

- Carbon, Ecosystems, \& Biogeochemistry including measurements of $\mathrm{CO} 2$ and $\mathrm{CH} 4$ fluxes and trends, vegetation 3D structure, biomass, and disturbance, ocean mixing layer

5.2 Mission Area II: Space Science and Exploration Focused Measurement Topic Areas

- Lunar Exploration including landing navigation and hazard avoidance, and surface exploration and characterization

- Jovian Moons including surface features and characterization, and tidal signatures

- Jovian Environment including atmospheric cloud and wind structure, atmospheric composition

- Mars Exploration including atmospheric winds, density, and dust profiling, surface topography, surface exploration and characterization, search for organic signatures, moisture distribution, and cloud characterization

- Martian Mission Support including precision landing, navigation, and hazard avoidance, sample return rendezvous and capture, atmospheric characterization to support entry, descent, and landing

- Titan and Venus including balloon-borne active optical instrumentation

- Situational awareness of space weather phenomena

- Laser interferometry, active -optical sensing of telescope and satellite swarm elements to enable observations

\subsection{Mission Area III: Aeronautics Focused Measurement Topic Areas}

- Turbulence detection on flight vehicles

- Situational awareness detection of atmospheric hazards including volcanic ash, ice, and snow

- Sense and avoidance for autonomous UAVs and future autonomous urban mobility aircraft

- Airport ground control management for taxiing, takeoff, and landing including wake vortex detection

- UAV-based target imaging

- Sensing and avoiding hazards in the National Air Space including miniaturized, low-power active optical sensors and instrumentation for UAV and personal air vehicles
- Quantification of flow field and surface parameters in ground-based wind tunnel testing of aero vehicle

To include the international active optical capability as a part of the NASA strategy, the S\&I CLT coordinated efforts with other space agencies and organized an International IEEE-GRSS Workshop on Space-based Lidar Remote Sensing Techniques and Emerging Technologies during June 4-8, 2018 at Milos Conference Center, Adamas, Milos Island, Cyclades, Greece. The NASA and ESA leadership jointly organized similar technical sessions as in the TIM. This Workshop brought together Active Optical experts and policy makers from NASA, ESA, JAXA, CNES, and DLR engaged in advancing space missions/measurements in the Active Optical area for Science and Exploration.

\section{CONCLUSIONS}

To address the critical Agency needs in the area of active optical measurements, the NASA Sensors and Instrumentation Capability Leadership Team worked with experts and leaders from NASA, industry, academia, FFRDCs, OGAs, and international Space Agencies to assess existing active optical capabilities and opportunities for leveraging and collaboration for addressing NASA's critical needs for Science, Exploration, and Aeronautics. The S\&I CLT hosted TIM and international workshop to capture national and international technical inputs from experts. Inputs gathered were provided to an independent and external Active Optical Assessment and Recommendation Team (AOART). In coming months, AOART team will complete a final report and present its findings, observation, and recommendation to the APMC, including a suggested strategy to address the Agency's needs in a crosscutting, synergistic and cost effective manner. The conference presentation will summarize these results and recommendations.

\section{REFERENCES}

[1] S. Alejandro, et al., NASA Internal Report, November 2000.

[2] U. Singh, et al., Proc. of SPIE 4882-81 (2002).

[3] F. Peri, et al., Proc. of SPIE 4893-24 (2002). 